\title{
The Development of Accommodation System to Faults of Navigation Sensors of Underwater Vehicles
}

\author{
Vladimir Filaretov, Alexey Zhirabok, Alexander Zuev, Alexander Protcenko \\ Far Eastern Federal University, Vladivostok, Russia, \\ Institute of Automation and Control Processes FEB RAS, Vladivostok, Russia.
}

\begin{abstract}
In this paper, a new approach for synthesis of the accommodation system to faults of navigation sensors of underwater vehicles (UV) is developed and investigated. Proposed system is synthesized by using kinematic model of UV and data fusion of sensors signals. The results of performed simulation have completely confirmed the working capacity and high quality of the proposed approach.
\end{abstract} faults

Keywords-diagnostic system; underwater vehicles; sensors;

\section{INTRODUCTION}

Today the development of different types of UV for fulfilment of underwater operations are very topical task. The main fields of UV using are the monitoring and maintenance of offshore structures or pipelines, the exploration of the sea bottom, fulfilment of underwater engineering operations and so on. Often UV should autonomously operate during long periods. Navigation sensors are one of the most important components of each $\mathrm{UV}$, which are necessary to motion control and navigation at performance of autonomous underwater missions. Obviously, the failure of navigation sensors will lead to erroneous mission fulfilment or loss of vehicles. Therefore, it is necessary to detect, isolate faulty sensor and estimate the value of fault as soon as possible. In case of faults arising, the diagnostic system should send information about detected faults to control system UV, which should decide to stop the mission or to continue by using special correction of control signals. The solution of this task is called by accommodation to faults.

There are several different synthesis methods of diagnostic and accommodation systems for UV [2-11]. These strategies use analytical model based techniques including Kalman filters, diagnostic (Luenberger like) observers, parity relations, neural network and so on. Analytical model based methods allow obtaining better performances of diagnostic procedure but need to use the sufficiently perfect mathematical models of vehicle dynamics. The characteristic feature of such models is that the equations of the vehicle motion are strongly nonlinear, coupled and has the variables and unknown parameters.

There are several approaches $[2,3]$ to robust diagnosing of UV. Their advantage is relative simplicity of the realization, however thus synthesized observers are capable to detect faults effectively only at horizontal movement of the UV with low speed.
In works [4-6], the sliding observers are proposed to use for formation of value of faults. Doubtless advantage of the given approaches is tolerance of the synthesized observers to unknown but slowly changing parameters of UV. However, the big problem interfering practical introduction of such systems is the problem of «chattering».

Hereby, problem of developing of effective accommodation system for navigation sensors of UV is important and topical. For solving this problem in paper is proposed new approach for synthesis of accommodation system for navigation sensors by using kinematic model of UV and data fusion of sensors signals. The special feedback for diagnostic observers is proposed for calculation of size of arising faults.

This system provides exact detection, isolation and compensation of faults in sensors of UV at performance of underwater missions in unknown environment with unknown external disturbances.

\section{DESCRIPTION OF UV MATHEMATICAL MODEL}

The dynamics of UV can be described by several sets of nonlinear differential equations. The precise mathematical models of UV are very complex and have the variable and unknown parameters. Therefore, diagnostic system synthesized on basis of these models cannot provide exact faults detection and isolation. At this case, it is possible to use kinematic model of UV for synthesizing of accommodation system to faults of navigation sensors.

In general, kinematic model of UV can be presented in matrix form [1]:

$$
\dot{\eta}=J(\eta) v
$$

where $\eta=[x, y, z, \varphi, \theta, \psi]^{T} \in R^{6}$ is a vector of position and orientation of $\mathrm{UV}$ in absolute coordinate system; $v=\left[v_{x}, v_{y}, v_{z}, \omega_{x}, \omega_{y}, \omega_{z}\right]^{T} \in R^{6}$ is a vector of projections of linear and angular velocities of UV on the axes of joined coordinate system; $\mathrm{T}$ is a symbol of transposition; $J(x)$ is the matrix of transition from jointed to absolute coordinate system which has a view [1]:

$$
J(\eta)=\left[\begin{array}{c:c}
J_{1}(\eta) & 0 \\
\hdashline 0 & J_{2}(\eta)
\end{array}\right]
$$


$J_{1}(\eta)=\left[\begin{array}{ccc}\cos \psi \cos \theta & -\sin \psi \cos \varphi- & \sin \psi \sin \varphi+ \\ & -\cos \psi \sin \theta \sin & +\cos \psi \cos \varphi \sin \theta \\ \sin \psi \cos \theta & \cos \psi \cos \varphi+ & -\cos \psi \sin \varphi+ \\ -\sin \theta & +\sin \varphi \sin \theta \sin \psi & +\sin \theta \sin \psi \cos \varphi \\ & \cos \theta \sin & \cos \theta \cos \varphi\end{array}\right]$,

$$
J_{2}(\eta)=\left[\begin{array}{ccc}
1 & \sin \varphi \tan \theta & \cos \varphi \tan \theta \\
0 & \cos \varphi & -\sin \varphi \\
0 & \frac{\sin }{\cos \theta} & \frac{\cos }{\cos \theta}
\end{array}\right] .
$$

The most underwater vehicles, intended for autonomous performance of various missions, have the following set of navigation sensors: 1) the Doppler speed $\log$, measuring linear speeds $\left(v_{x}, v_{y}, v_{z}\right)$ of UV in joint coordinate system; 2 ) the sensors of angular speeds of UV $\left(\omega_{x}, \omega_{y}, \omega_{z}\right)$ concerning joint coordinate system;

3 ) the sensor of orientation of UV $(\varphi, \theta, \psi)$, measuring angles of heel, trim and course in absolute coordinate system; 4) the hydroacoustic navigation system (HNS), measuring linear coordinates of UVs $(x, y)$ in absolute coordinate system;

5 ) the sensor of depth of UV (z), measuring coordinate of $z$ in absolute coordinate system.

Thus sensors measure all components of vectors of $v$ and $\eta$ in kinematic model of UV (see equation 1). At this, the faults $d v$ and $d \eta$ can be arising in these sensors:

$$
\tilde{\eta}=\eta+d \eta, \quad \tilde{v}=v+d v,
$$

where $d v=\left[d v_{x}, d v_{y}, d v_{z}, d \omega_{x}, d \omega_{y}, d \omega_{z}\right]^{\mathrm{T}}, d \eta=[d x, d y$, $d z, d \varphi, d \theta, d \psi]^{\mathrm{T}} ; \widetilde{\eta}, \widetilde{v}$ are output signals of navigation sensors with possible faults.

In case faults are absent, all elements of vectors of $d v$ and $d \eta$ are equal to zero.

There are many different causes leading to the sensors fault. The faulty components should be early detected and isolated by the diagnostic system to avoid the erroneous mission fulfilment or loss of the UV.

\section{SYNTHESIS OF DIAGNOSTIC OBSERVERS}

The formation of residuals consists in producing a signal, which carries information about the faults. For each sensor, the particular observer to generate the residual will be used. Diagnosing is carried out by means of check of algebraic ratios to which have to satisfy output signals of navigation sensors and observers in the absence of faults. Vectors of output signals of sensors $\tilde{\eta}$ and observers $\bar{\eta}$ are compared therefore the vector of residual $r$ is formed (at the absence of faults $r=0$ ).

Let's introduce the observers $O_{1}-O_{6}$, which model the signals $x, y, z, \varphi, \theta, \psi$ accordingly and are described by equations similar to (1):

$$
\dot{\bar{\eta}}=J(\tilde{\eta}) \tilde{v} .
$$

At this, the residual vector $r=\left[r_{1}, r_{2}, r_{3}, r_{4}, r_{5}, r_{6}\right]^{\mathrm{T}}$ is formed as:

$$
r=\tilde{\eta}-\bar{\eta} .
$$

If we rewrite the (3) by taking into account equation (2) we can obtain the equations for observers:

$$
\begin{aligned}
& \dot{\bar{x}}=J_{11} \widetilde{v}_{x}+J_{12} \widetilde{v}_{y}+J_{13} \widetilde{v}_{z}, \dot{\bar{y}}=J_{21} \widetilde{v}_{x}+J_{22} \widetilde{v}_{y}+J_{23} \widetilde{v}_{z}, \\
& \dot{\bar{z}}=J_{31} \widetilde{v}_{x}+J_{32} \widetilde{v}_{y}+J_{33} \widetilde{v}_{z}, \dot{\bar{\varphi}}=J_{44} \widetilde{\omega}_{x}+J_{45} \widetilde{\omega}_{y}+J_{46} \widetilde{\omega}_{z},(5) \\
& \dot{\bar{\theta}}=J_{55} \widetilde{\omega}_{y}+J_{56} \widetilde{\omega}_{z}, \dot{\bar{\psi}}=J_{65} \widetilde{\omega}_{y}+J_{66} \widetilde{\omega}_{z},
\end{aligned}
$$

where $J_{i j}$ - elements of the matrix $J$.

To isolate the faults we need to designate the relations between faults and residuals. These relations can be represented in the form of the faults matrix $M_{f}$ (see Table 1). The rows of this matrix correspond to the residuals and columns correspond to the faults in the components. The values of the faults matrix are 0 and 1 which reflect a fact of sensitivity of residuals to the fault in some component. If residual is sensitive to particular fault, the intersection of corresponding row and column of this matrix is indicated by the value 1 ; otherwise 0 . In our case, the faults matrix $M_{f}$ will have the following form:

Table I. Faults Matrix $M_{f}$.

\begin{tabular}{|l|l|l|l|l|l|l|l|l|l|l|l|l|}
\hline & $d v_{x}$ & $d v_{y}$ & $d v_{z}$ & $d \omega_{x}$ & $d \omega_{y}$ & $d \omega_{z}$ & $d x$ & $d y$ & $d z$ & $d \varphi$ & $d \psi$ & $d \theta$ \\
\hline$r_{1}$ & 1 & 1 & 1 & 0 & 0 & 0 & 1 & 0 & 0 & 1 & 1 & 1 \\
\hline$r_{2}$ & 1 & 1 & 1 & 0 & 0 & 0 & 0 & 1 & 0 & 1 & 1 & 1 \\
\hline$r_{3}$ & 1 & 1 & 1 & 0 & 0 & 0 & 0 & 0 & 1 & 1 & 0 & 1 \\
\hline$r_{4}$ & 0 & 0 & 0 & 1 & 0 & 0 & 0 & 0 & 0 & 1 & 0 & 1 \\
\hline$r_{5}$ & 0 & 0 & 0 & 1 & 1 & 1 & 0 & 0 & 0 & 1 & 1 & 0 \\
\hline$r_{6}$ & 0 & 0 & 0 & 1 & 1 & 1 & 0 & 0 & 0 & 1 & 0 & 1 \\
\hline
\end{tabular}

To isolate faulty sensor we need compare the results of residual evaluation with signatures of particular faults. The signature of the $i$-th fault is the $i$-th column of the faults matrix $M_{f}$. If they coincidence, this means that the fault did occurs in correspond UV sensor. As follows from the faults matrix $M_{f}$, the faults $d v_{x}, d v_{y}, d v_{z}$ and $d \omega_{y}$ are not distinguished from one another because its signatures are similar. Therefore, the faulty sensor cannot be isolated correctly by the diagnostic system. To improve the fault isolation property, the additional observers are suggested.

Let's express from first, second, third and fifth equations of (5) the following values:

$$
\begin{aligned}
& \widetilde{v}_{x}=\frac{\dot{\bar{x}}-\left(J_{12} \tilde{v}_{y}+J_{13} \tilde{v}_{z}\right)}{J_{11}}, \widetilde{v}_{y}=\frac{\dot{\bar{y}}-\left(J_{21} \widetilde{v}_{x}+J_{23} \widetilde{v}_{z}\right)}{J_{22}}, \\
& \widetilde{v}_{z}=\frac{\dot{\bar{z}}-\left(J_{31} \widetilde{v}_{x}+J_{32} \widetilde{v}_{y}\right)}{J_{33}}, \widetilde{\omega}_{y}=\frac{\dot{\bar{\theta}}-J_{56} \bar{\omega}_{z}}{J_{55}},
\end{aligned}
$$


and substitute $\widetilde{v}_{x}$ into $O_{3}, \widetilde{v}_{y}$ into $O_{1}, \widetilde{v}_{z}$ into $O_{2}$ and $\widetilde{\omega}_{y}$ into $O_{4}$. As a result, it is possible to obtain the additional observers $\mathrm{O}_{7}-O_{10}$, residuals of which did not depend from $d v_{y}, d v_{z}, d v_{x}$ and $d \omega_{y}$ accordingly. Therefore it is possible to unambiguous define the faulty sensor.

\section{ESTIMATION OF MAGNITUDE OF FAULTS}

After detecting of faults, which occurring in the sensors, it is necessary accurately identify magnitude of these faults in real time. To calculate the faults $d v_{x} \ldots, d \omega_{z}$, observers $O S_{1}-O S_{6}$ is introduced. They are described by the system of equations of the form identical to the system (5): $\dot{\hat{\eta}}=J(\tilde{\eta}) \tilde{v}, \hat{r}=\tilde{\eta}-\hat{\eta}$. Consider the observer $O S_{1}$, which determines the magnitude of the fault $d v_{x}$. It is described by the following equation: $\dot{\hat{x}}=J_{11}\left(v_{x}+d v_{x}\right)+J_{12} v_{y}+J_{13} v_{z}$.

Next, it is necessary to introduce into the observer feedback by residual with a gain of $w$ : $\dot{\hat{x}}=J_{11}\left(v_{x}+d v_{x}\right)+J_{12} v_{y}+J_{13} v_{z}+w \hat{r}_{1}$. With taking into account expression (4) it's possible to obtain:

$\dot{\hat{r}}=\dot{\tilde{x}}-J_{11}\left(v_{x}+d v_{x}\right)-J_{12} v_{y}-J_{13} v_{z}-w \hat{r}_{1}, \dot{\hat{r}}_{1}=-J_{11} d v_{x}-w \hat{r}_{1}$

Suppose that the size of the $d v_{x}(t)$ is variable and can be represented as: $d v_{x}(t)=a e^{j \omega t}$, where $a$ - the amplitude of error; $\omega$ - frequency at which faults change; $j$ - the imaginary unit. In this case, the solution of equation (7) has the form:

$$
\hat{r}_{1}(t)=C_{1} e^{-w t}+\frac{-J_{11} a}{\sqrt{w^{2}+\omega^{2}}} e^{j \omega t+\phi}
$$

where $\phi=\operatorname{actg}(\omega / w), C_{1}$ - constant.

If it is assumed that frequency is much smaller than the feedback gain $\omega<<w$ then (8) reduces to the following:

$$
\hat{r}_{1}(t)=C_{1} e^{-w t}-\frac{J_{11} d v_{x}}{w} .
$$

From (9) it follows that the value of $w$ determines the speed of the transition process. It is known, that it is desirable to choose $w=3 / T_{C}$, where $T_{C}$ - the duration of the transition process. After the end of the transition process, the size of the fault can be obtained from the following expression: $d v_{x}=-\frac{\hat{r}_{1}(t) w}{J_{11}}$.

Feedback is introduced for observers $\mathrm{OS}_{2}-\mathrm{OS}_{6}$ in similar manner.

To calculate the fault $d x, d y, d z, d \psi$, observers $O S_{7}$, $O S_{8}, O S_{9}, O S_{11}$ are introduced. They are described by the $1 \mathrm{st}, 2 \mathrm{nd}, 3 \mathrm{rd}$ and 5 th equations from system. Consider the observer $\mathrm{OS}_{7}$, which determines the magnitude of the fault $d x$. It is described by the following equation: $\dot{\hat{x}}=J_{11} v_{x}+J_{12} v_{y}+J_{13} v_{z}$. In the event of a fault residual of the observer becomes $\dot{\hat{r}}_{7}=\dot{\vec{x}}-J_{11} v_{x}-J_{12} v_{y}-J_{13} v_{z}$. With taking into account expression (4) we can receive $\dot{\hat{r}}_{7}=\dot{x}+d \dot{x}-J_{11} v_{x}-J_{12} v_{y}-J_{13} v_{z}, \quad \dot{\hat{r}}_{7}=d \dot{x}$. Hence, it follows that $\hat{r}_{7}=d x$.

Thus, the sought-for size of fault is estimated. This is also true for observers $O S_{8}, O S_{9}, O S_{11}$.

Next, observer $O S_{10}$ is introduced to calculate the fault $d \varphi$. For this, it is necessary to solve the system of 5 th and 6th equations of system (5):

$$
\left\{\begin{array}{l}
\dot{\widetilde{\theta}}=(\cos \varphi) \widetilde{\omega}_{y}-(\sin \varphi) \widetilde{\omega}_{z}, \\
\dot{\widetilde{\psi}}=\left(\frac{\sin \varphi}{\cos \widetilde{\theta}}\right) \widetilde{\omega}_{y}+\left(\frac{\cos \varphi}{\cos \widetilde{\theta}}\right) \widetilde{\omega}_{z} .
\end{array}\right.
$$

Solution can be found as follows:

$$
\varphi=\arcsin \frac{\widetilde{\omega}_{y} \dot{\widetilde{\psi}} \cos \tilde{\theta}-\widetilde{\omega}_{z} \dot{\tilde{\theta}}}{\widetilde{\omega}_{y}{ }^{2}+\widetilde{\omega}_{z}{ }^{2}} .
$$

The residual in this case will be $\hat{r}_{10}=\widetilde{\varphi}-\varphi=d \varphi$.

The observer $O S_{12}$ (for fault $d \theta$ ), is searched in similar manner from the 1 st and 2 nd equations of the system (5).

Thus at the output of observers $O S_{1}-O S_{12}$, we obtain a set of values of the magnitudes of faults.

The developed method allows to determine, locate and calculate the size of the faults that occur in the navigation sensors of UV. Using these values of real signals from sensors in control system it's possible to synthesize accomodation system.

\section{SiMULATION OF ACCOMMODATION SYSTEM}

To investigate the effectiveness of the proposed method of synthesis of accommodation systems, simulations were performed. During the simulation UV carried movement along a predetermined path in the XY plane. The simulation was done with zero initial conditions of UV and observers. Faults in the sensors were simulated by adding a $d v_{v}=0,5 \mathrm{~m} / \mathrm{s}$ to sensor signal which began at time $t_{f}=12 \mathrm{~s}$. Fig. 1 shows UV trajectory in the absence of faults, when fault is present and the fault compensated by accommodation system. 


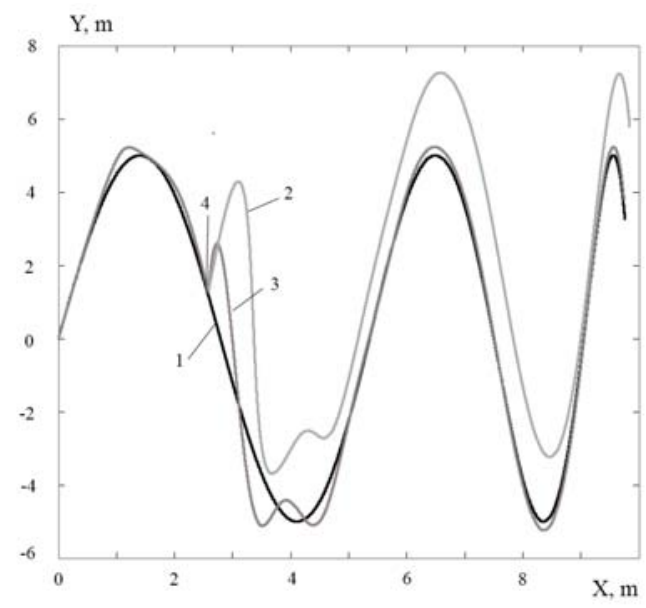

Figure 1. UV trajectory in the XY plane. 1 - specified trajectory, 2 trajectory when fault is present, 3 - trajectory with the accommodation system, 4 - point of introduction of fault.

As can be seen from the fig. 1, synthesized system of accommodation provide the compensation of arising fault. Similar results were obtained in the simulation cases of other types of faults. Thus, the results of mathematical modelling is fully confirmed the efficiency and high performance of the proposed method of synthesis of accommodation systems for typical faults in the navigation sensors of UVs.

\section{CONCLUSION}

In this paper, an effective method for the synthesis of high-quality systems of accommodation to frequently occurring types of faults in the navigation sensors of UV is proposed. Developed system are synthesized by using kinematic model of UV and data fusion of sensors signals and allows detecting and isolating faults in sensors of UV at performance of underwater missions in unknown environment with unknown external disturbances.

The simulation of the obtained systems confirmed the high quality of the diagnosis procedure and accommodation. It allows to identify and locate faults in these devices well-timed, operatively fend off their effects, to prevent the occurrence of extraordinary events, stopping execution process, the failure of the mission, or loss of UV. This problem was solved with the help of method of accommodation to faults. These systems provide invariance properties, by detecting faults of sensors and estimate the true values of the measured variables, which guarantees the continued operation and effectiveness of UV during their operation.

\section{ACKNOWLEDGMENT}

Research is executed at the expense of a grant of the Russian scientific fund (project No. 14-19-00081).

\section{REFERENCES}

[1] Thor I. Fossen. Guidance and control of ocean vehicles // University of Trondheim. Norway. 1995.

[2] Alessandri A., Caccia M., Verruggio G. Fault Detection of Actuator Faults in Unmanned Underwater Vehicles // Control Engineering Practice. V. 7. 1999. P. 357-368.

[3] Alessandri A., Caccia M., Verruggio G. A Model-Based Approach to Fault Diagnosis in Unmanned Underwater Vehicles // Oceans. Nice, F. 1998. P. 825-829.

[4] Alessandri A., Hawkinson T., Healey A.J., Veruggio G. Robust Model-Based Fault Diagnosis for Unmanned Underwater Vehicles Using Sliding Mode Observers // Proc. Int. Symposium Unmanned Untethered Submersible Technology, August 1999.

[5] Dhahri S., Ben Hmida F., Sellami A. LMI-based sliding-mode observer design method for reconstruction of actuator and sensor faults // Int. Journal on Sciences and Techniques of Automatic control. V. 1. №1. 2007. P. 91-107.

[6] Hakiki K., Mazari B., Liaizid A., Djaber S. Fault Reconstruction Using Sliding Mode Observers // American Journal of Applied Sciences. 2006. P. 1669-1674. (6.)

[7] Filaretov V., Zhirabok A., Kucher D. "New approach to robust observer design", Proc. 19-th Intern. DAAAM Symposium, Slovakia, October, 2008, pp.491-492.

[8] Join C., Ponsart J-C., Sauter D. Sufficient conditions to fault isolation in nonlinear systems: a geometric approach // CD ROM Proc. 15th IFAC World Congr. Automat. Control. Barcelona, Spain, 2002.

[9] Frank P. Fault diagnosis in dynamic systems using analytical and knowledge-based redundancy. A survey and some new results // Automatica. 1990. V. 26. P. 459-474.

[10]Deuker B., Perrier M., Amy B. Fault-Diagnosis of Subsea Robots Using Neuro-Symbolic Hybrid Systems // Oceans. Nice, F. 1998. P. 830-834.

[11] Farrell J., Berger T., Appleby B.D. Using Learning Techniques to Accomodate Unanticipated Faults // Control Systems Magazine. V. 13, №. 3. P. 40-49. 